\title{
Dynamic separation on a pitching and surging airfoil as a model for flow over vertical axis wind turbine blades
}

\author{
R. Dunne ${ }^{*}$ and B.J. McKeon ${ }^{\dagger}$ \\ California Institute of Technology, Pasadena, CA, USA
}

\begin{abstract}
Vertical axis wind turbine (VAWT) blades undergo dynamic separation due to the large angle of attack variation they experience during a turbine rotation. The flow over a single blade was modeled using a sinusoidally pitching and surging airfoil in a constant free stream flow at a mean chord Reynolds number of $10^{5}$. Two-dimensional, time resolved velocity fields were acquired using particle image velocimetry (PIV). Vorticity contours were used to visualize shear layer and vortex activity. A low order model of dynamic separation was developed using Dynamic Mode Decomposition (DMD). A primary and secondary dynamic separation mode were identified as the critical drivers for the unsteady flow field.
\end{abstract}

\section{Nomenclature}

$\mathrm{t} \quad$ time $[\mathrm{s}]$

$\alpha \quad$ Angle of attack [ $\left.{ }^{\circ}\right]$

$\theta \quad$ Turbine rotation angle $\left[^{\circ}\right]$

Re Chord Reynolds number $\left(\frac{\mathrm{Uc}}{\nu}\right)$

$\mathrm{U}_{\infty}$ Windspeed $[\mathrm{m} / \mathrm{s}]$

U Relative velocity to VAWT blade $[\mathrm{m} / \mathrm{s}$ ]

$\overline{\mathrm{U}}$ Average velocity over turbine rotation $[\mathrm{m} / \mathrm{s}]$

$\eta \quad$ Tip speed ratio $\left(\frac{\omega \mathrm{R}}{\mathrm{U}_{\infty}}\right)$

$\omega \quad$ Turbine frequency $[\mathrm{rad} / \mathrm{s}]$

$\Omega \quad$ Pitch surge frequency [rad/s]

$\mathrm{R} \quad$ Turbine radius $[\mathrm{m}]$

Ro Rossby number $\left(\frac{\mathrm{U}_{\infty}}{2 \mathrm{c} \omega}\right)$

c Chord length $[\mathrm{cm}]$

$\nu \quad$ Kinematic viscosity $\left[\mathrm{m}^{2} / \mathrm{s}\right]$

$\lambda$ Transformed DMD eigenvalues

$\mathrm{k} \quad$ Reduced frequency

$\Gamma_{1} \quad$ Vortex center detection algorithm

$\Gamma_{2} \quad$ Vortex boundary detection algorithm

$\mu_{\Gamma} \quad$ Vortex circulation $\left[\mathrm{m}^{2} / \mathrm{s}\right]$

$\mathrm{x} \quad$ Streamwise coordinate $[\mathrm{c}]$

y Cross-stream coordinate [c]

$\mathrm{xl}$ Leading edge position [c]

subscript

$i \quad$ Imaginary component

$r \quad$ Real component

$j \quad$ Variable number

\footnotetext{
*Graduate Student, Mechanical Engineering, California Institute of Technology, Pasadena, CA, 91125, AIAA Student Member.

${ }^{\dagger}$ Professor, Graduate Aerospace Laboratories California Institute of Technology, Pasadena, CA, 91125, AIAA Associate Fellow.
} 


\section{Introduction}

$W^{\text {IND }}$ is growing as a major contributor to energy production in the United States representing $3.6 \%$ of the total in 2012. ${ }^{1}$ Most of this energy is currently produced using large scale horizontal axis wind turbines (HAWTs). While individual HAWTs are very efficient, power output from wind farms is limited by turbine spacing, with significant aerodynamic interference present between turbines spaced less than 20 rotor diameters apart. To achieve $90 \%$ of peak efficiency from each turbine HAWTs must be separated by $8-10$ diameters in the downstream direction and 3-5 in the cross stream direction. ${ }^{2}$ Recent studies have shown that vertical axis wind turbines (VAWTs) can be placed much closer together to achieve a potential order of magnitude increase in energy extracted from a given plot of land. ${ }^{3}$ Additionally VAWTs are insensitive to wind direction, quieter, simpler in design and construction with fewer moving parts and often a constant blade profile along the span. ${ }^{4,5}$

Typical VAWTs operate at tip speed ratios, defined as the blade speed divided by the incoming windspeed $\left(\eta=\frac{\omega \mathrm{R}}{\mathrm{U}_{\infty}}\right)$, of between 2 and 3 where $\mathrm{R}$ is the radius of the turbine, $\mathrm{U}_{\infty}$ is the free stream wind velocity and $\omega$ the turbine rotation rate. Due to the perpendicular incoming flow and turbine rotation axis, a single blade of a VAWT goes through a periodic change in angle of attack and relative velocity during the turbine cycle. A schematic of a typical VAWT is shown in figure 1 demonstrating angle of attack $\alpha$ and velocity U. At low tip speed ratios this angle of attack variation drives the airfoil well above its static stall angle resulting in dynamic stall on each blade twice per turbine cycle, once on each side of the blade (figure 2). Dynamic stall causes an abrupt drop in the lift of the blade, and therefore torque on the turbine, as well as potentially damaging unsteady loading on the generator and turbine structure. ${ }^{4}$ Error in predicting these dynamic loads can decrease VAWT lifetimes by a factor of up to 70. Thus a complete understanding of the flow field is required for optimal VAWT design. ${ }^{6}$

Dynamic stall on a representative one-bladed VAWT has been studied experimentally and computationally at Reynolds numbers near operating conditions and tip speed ratios of 2,3 and 4 by Ferreira et al..$^{7-10}$ The growth of leading edge and trailing edge vorticity was analysed at several positions around the turbine cycle and the total vortex circulation was shown to grow until the vortex is shed at the point of dynamic stall. Additionally the forces on the turbine blades and the associated torque on the turbine were computed in the simulations. Experiments performed by Jones and Babinsky ${ }^{11}$ on rotating wings at $\mathrm{Re}=60,000$ showed significant unsteady lift increases during leading edge vorticity growth followed by a decrease below the steady value after the separation of this leading edge vortex. PIV analysis of Mulleners and Raffel ${ }^{12,13}$ on an airfoil pitching around a statically attached angle of attack identified a 'primary stall vortex' pinched off at the point of dynamic stall.

This work explores the flow over a sinusoidally pitching and surging airfoil as a surrogate for the blade of a vertical axis wind turbine. Comparison of the turbine kinematics to the sinusoidal pitch/surge motion used in the experiment is shown in figure 2. Pitching and surging motion can capture the angle of attack and velocity variation of the turbine, however it neglects the the Coriolis effect due to the rotation of the turbine. In the linear, experimental frame, the Coriolis effect imposes a force on the flow to account for the curved path of the turbine blade. The relative importance of this Coriolis force in a VAWT can be measured using the ratio of inertial to rotational forces, the Rossby number $\left(\operatorname{Ro}_{0}=\frac{\mathrm{U}_{\infty}}{2 * c * \omega}\right)$. Using the definition of the tip speed ratio $\left(\eta=\frac{\omega \mathrm{R}}{\mathrm{U}_{\infty}}\right)$ simplifies the Rossby number to be dependent only on the geometry and operating condition of the turbine, $\mathrm{Ro}=\frac{\mathrm{R}}{2 * \mathrm{c} * \eta}$. For a standard industrial turbine at $\eta=2, c=15 \mathrm{~cm}$ the Rossby number is order 1 suggesting that rotational effects need consideration in addition to the pitch surge dynamics. ${ }^{14}$ Working with simplified linear pitch/surge motion however makes it possible to separate the pitching and surging effects. Additionally it permits time resolved particle image velocimetry (PIV) measurements to be taken over the entire effective turbine rotation. These time resolved measurements provide insight into the evolution of dynamic separation and vortical structure on a single turbine blade. Dynamic mode decomposition (DMD) as developed by Schmid ${ }^{15}$ was used on the time series to isolate and rank periodic structures by their dynamic significance. A model of the flow using three of the strongest modes was able to capture the dynamic separation and reattachment of the flow. Analysis of the individual modes identified in this model was used to investigate structure inherent in the flow. 


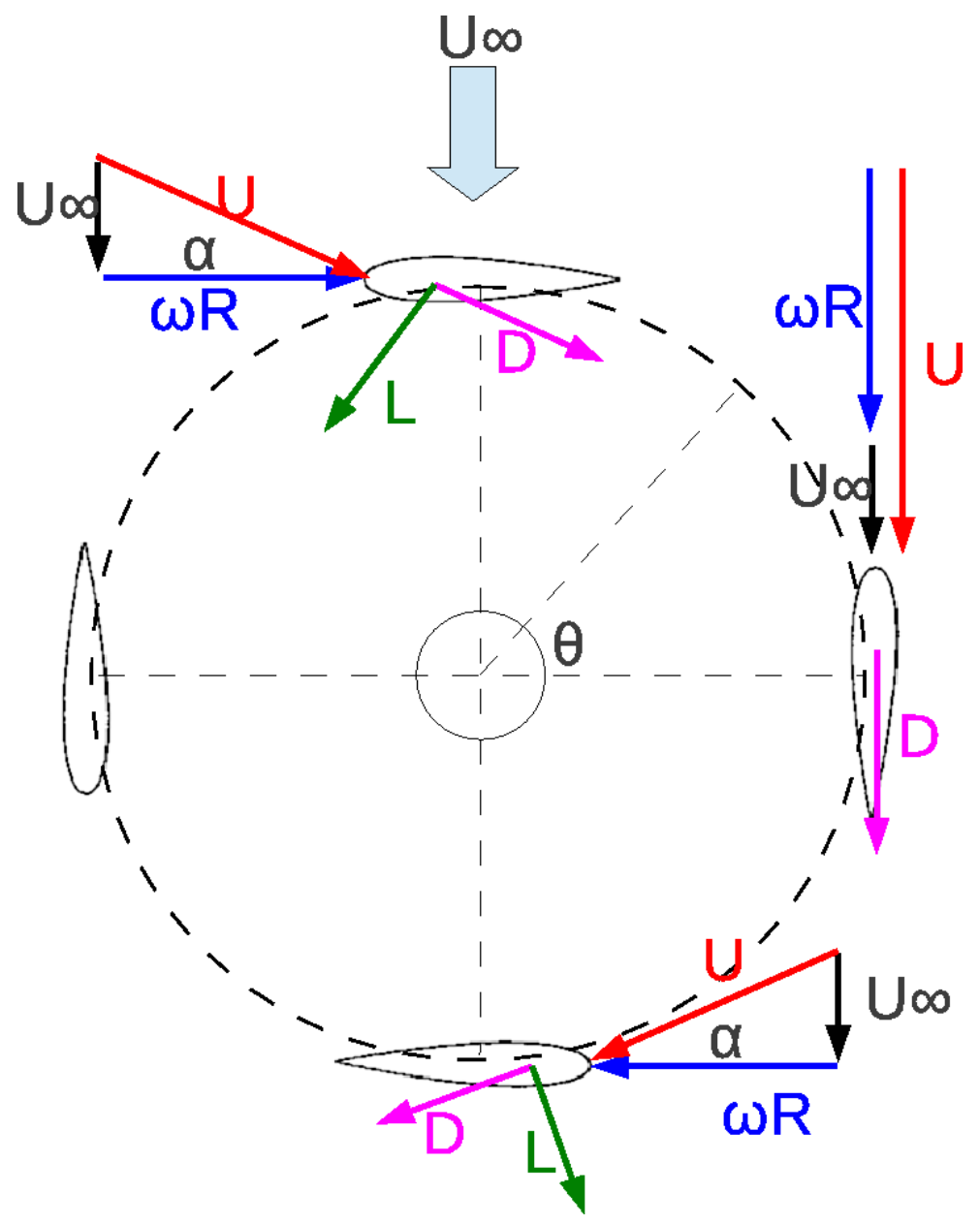

Figure 1: Top view of a typical VAWT. Wind speed $\mathrm{U}_{\infty}$ (black), relative velocity $\mathrm{U}$ (red), blade velocity $\omega R$ (blue), L,D lift and drag directions (green and magenta).
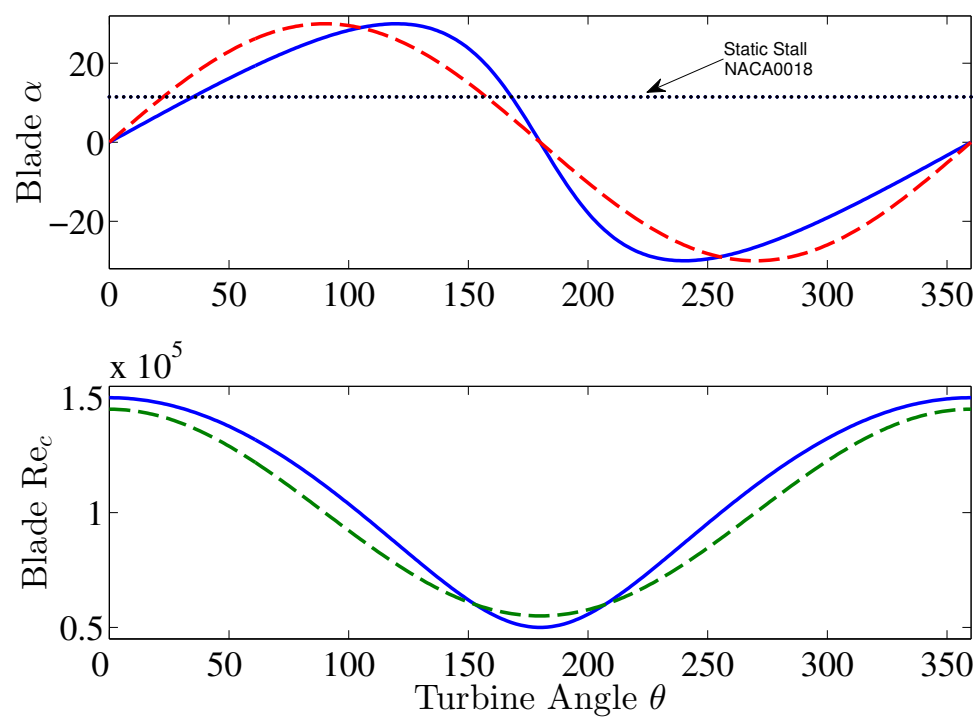

Figure 2: Periodic velocity and Reynolds number variation over typical VAWT blade, for $\eta=2, U_{\infty}=5 \mathrm{~m}$ $\mathrm{s}^{-1}, \mathrm{c}=15 \mathrm{~cm}$ (solid lines). Compared to test motion (dashed lines). 


\section{Experimental Setup}

\section{A. Hardware}

A 200mm chord NACA 0018 airfoil was constructed out of acrylonitrile butadiene styrene (ABS) plastic by fused deposition modeling (FDM), painted black and sanded smooth to minimize reflection from the PIV laser. The symmetrical NACA 0018 was chosen such that the flow on both pressure and suction side of the airfoil could be investigated in a single cycle. It is thick enough to be easily mounted in the water tunnel with negligible deflection along the span during high load. Additionally it is a well characterized airfoil, used in multiple VAWT studies (as well as thinner airfoils of the same family NACA 0012-0015). ${ }^{5,7-10}$ It is suspended above a $1 \mathrm{~m}$ wide $\mathrm{x} 1.5 \mathrm{~m}$ long, $0.5 \mathrm{~m}$ deep recirculating water channel capable of velocities up to $70 \mathrm{~cm} / \mathrm{s}$. The tip of the airfoil comes within $1 \mathrm{~mm}$ of the bottom of the tunnel to minimize wall effects. A $12.7 \mathrm{~mm}$ shaft is attached to the airfoil at the leading edge, supported by a thrust bearing, and directly connects to a NEMA 34-485 microstepping 2-phase motor with holding torque of $3200 \mathrm{~N}-\mathrm{mm}$. This results in a dynamic pitch system with $1 / 20,000$ of a rotation $\left(0.018^{\circ}\right)$ accuracy, and zero backlash. The motor and thrust bearing are fixed to a small $300 \mathrm{~mm}$ square by $200 \mathrm{~mm}$ deep aluminium cart mounted to linear bearings supported on rails outside the water channel, figure 3 . A $19 \mathrm{~mm}$ ball nut is attached to the cart and actuated on a $1.22 \mathrm{~m}$ long $19 \mathrm{~mm}$ diameter ballscrew with backlash of $\leq 0.2 \mathrm{~mm}$. This ball screw assembly is attached to a NEMA 34-490 microstepping motor with holding torque of $9900 \mathrm{~N}-\mathrm{m}$ resulting in linear position control system with $0.00064 \mathrm{~mm}$ accuracy. Both pitch and surge control systems were measured using 2000 step optical encoders to further ensure accuracy and repeatability of the experiments. Control and measurement of angle of attack (pitch) and linear position (surge) was performed simultaneously using National Instruments LabVIEW ${ }^{\mathrm{TM}}$ and a National Instruments PCIe-6321 data acquisition card.

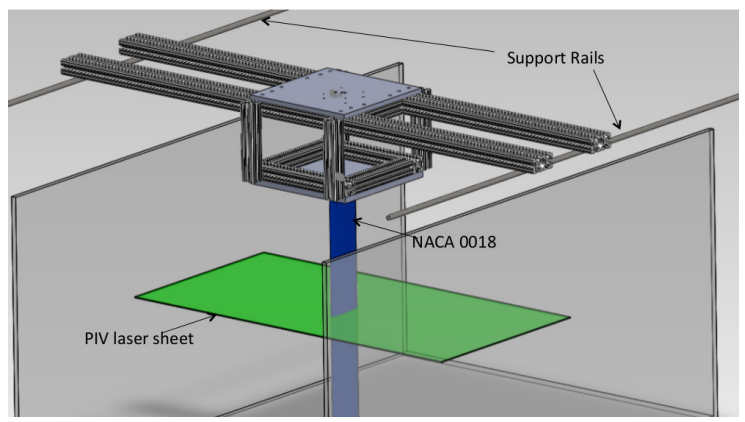

(a) Schematic of experimental apparatus.

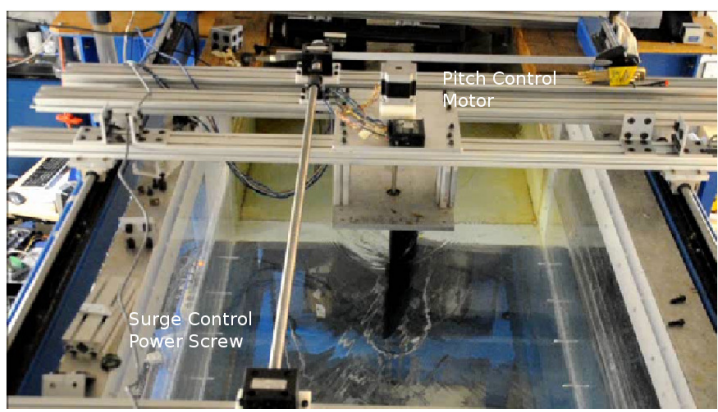

(b) Picture of apparatus installed in water channel. Flow from bottom to top.

Figure 3: Experimental pitch/surge mechanism.

Time resolved velocity measurements were made in the streamwise, airfoil-normal $(\mathrm{x}, \mathrm{y})$ plane approximately $40 \%$ of the span above the water channel floor using a commercial LaVision PIV system. Well mixed, neutrally buoyant, hollow, silver coated, glass spheres approximately $100 \mu \mathrm{m}$ in diameter were used for PIV seeding, illuminated by a steady 25mJ DM20-527 Photonics YAG laser. Two Photron Fastcam APS-RX cameras, aligned in the wall normal and offset in the streamwise direction with some overlap, were used to capture 2048 sequential images with 1024 X 1024 pixel resolution yielding a field of view of $69 \mathrm{X} 31 \mathrm{~cm}(3.5$ $X$ 1.5c) (streamwise $X$ airfoil-normal). Images were taken at $80 \mathrm{~Hz}$ with an exposure time of $1 / 400$ s yielding 2047 velocity fields with a duration of $25.6 \mathrm{~s}$. Velocity vectors were calculated using La Vision Davis software, and velocity fields from different cameras were knitted together in post processing. Three vector processing runs were perfomed with a 50\% window overlap and decreasing interrogation window of 64 pixels for the first run and 32 for the final two. The final data was smoothed in space using a $3 \times 3$ filter. This process yields a spatial resolution of $5.5 \times 5.5 \mathrm{~mm}(.028 \mathrm{X} .028 \mathrm{c})$;

In order to attain a velocity field over the entire airfoil pitch-surge cycle two separate experiments were performed on an overlapping front and back field of view. Twenty experiments were performed in each field of view with airfoil position $(\mathrm{xl}, \alpha)$ of the airfoil carefully measured during the entire experiment (where $\mathrm{xl}=\mathrm{xl}(\mathrm{t})$ is the time varying position of leading edge of the airfoil in laboratory reference frame due to surge motion). Datasets with measured leading edge position xl within $1 \%$ and angle of attack $\alpha$ within $0.5^{\circ}$ of 
desired position were phase averaged together based on airfoil position. This resulted in 15-18 individual experiments included in the phase averaged velocity data for each field of view. Both fields of view were then knit together to form a near complete picture of the flow. Figure 4a shows the position of the airfoil in the field of view at its maximum aft and forward position. This composite field of view measures the flow over the leading edge of the airfoil for its entire cycle. As a final post processing step the velocity fields were rotated into the airfoil reference frame with the center of the leading edge at the origin with positive $\mathrm{x}$ in the upstream direction. White lines in figure $4 \mathrm{~b}$ show the position of the rotated field of view over the airfoil where time has been extruded along the $\mathrm{Z}$ axis. Further experiments are necessary to examine the trailing edge at the furthest aft point in the cycle. Data is only presented on the top surface of the airfoil as the shadow of the airfoil prevents PIV vector calculation on the bottom surface (figure 4a).
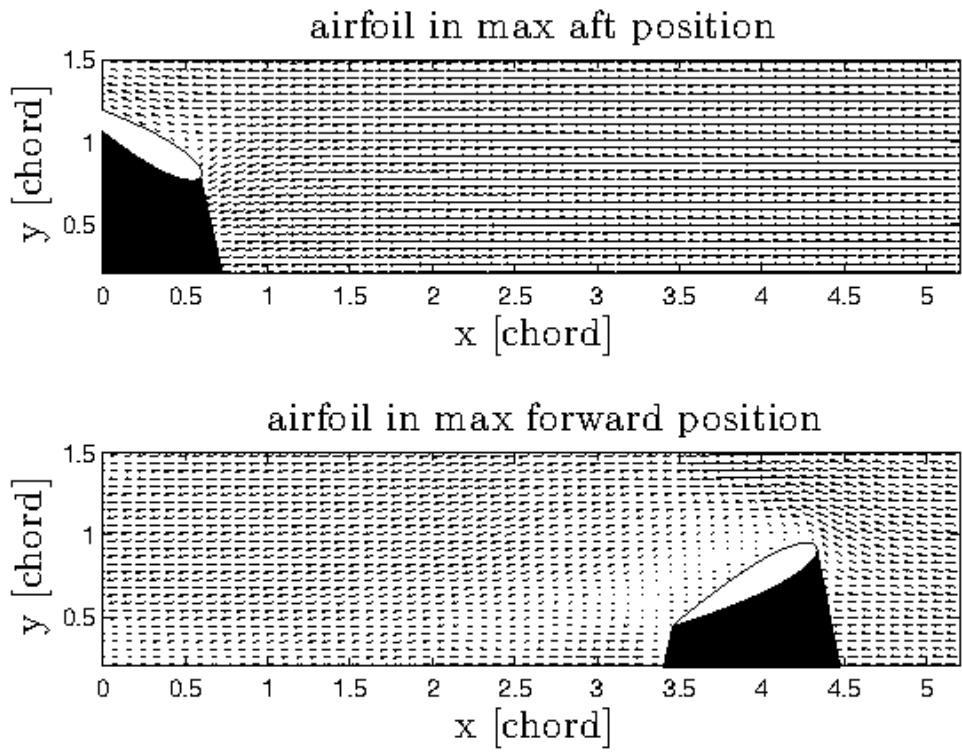

(a) Vector plot over airfoil at maximum and minimum streamwise position $\mathrm{x}$. Incoming flow right to left, laser illumination from above, black area below airfoil represents shadow where vectors cannot be calculated.

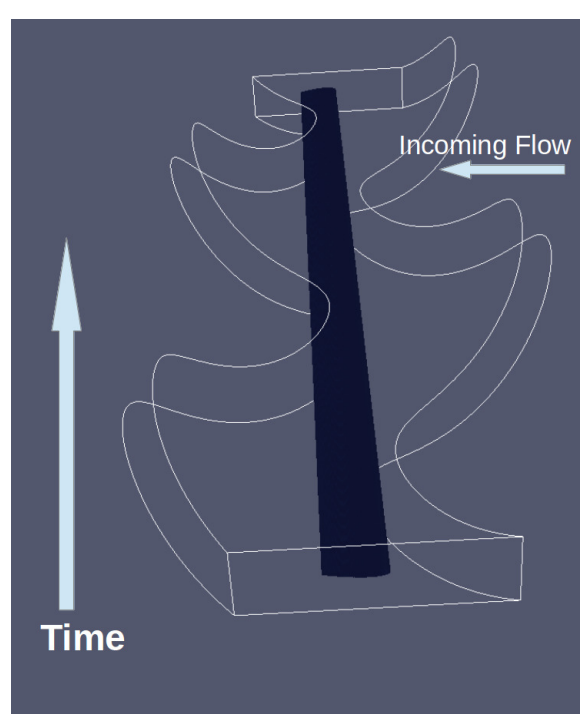

(b) field of view in airfoil frame. White lines show boundary of experimental field of view in airfoil reference frame. Flow right to left.

Figure 4: Field of view in experimental and airfoil fixed frame.

\section{B. Airfoil Dynamics}

Experimental parameters including Reynolds number, pitch and surge amplitude, frequency and phase were chosen to closely mimic that of a representative $\eta=2$ vertical axis wind turbine in the American Wind Energy Association (AWEA) national average wind velocity of $5 \mathrm{~m} \mathrm{~s}^{-1} \cdot{ }^{16}$ A mean chord Reynolds number of $10^{5}$ was achieved in room temperature water with kinematic viscosity $\nu=10^{-6} \mathrm{~m}^{2} \mathrm{~s}^{-1}$ and tunnel velocity $\overline{\mathrm{U}}=50 \mathrm{~cm} \mathrm{~s}^{-1}$. Sinusoidal pitch between -30 and $30^{\circ}$ about the leading edge and surge of $\frac{\mathrm{U}_{\max }-\mathrm{U}_{\min }}{\mathrm{U}}=.9$ were chosen to match the angle of attack and Reynolds number variation of the turbine shown in figure 2 . A pitch and surge frequency of $\Omega=.6 \mathrm{rad} \mathrm{s}^{-1}$ was chosen based on the reduced frequency of the turbine $\mathrm{k}=\frac{\omega \mathrm{c}}{2 \eta \mathrm{U}_{\infty}}=0.12$.

\section{The Dynamic Mode Decomposition}

The Dynamic Mode Decomposition ${ }^{15}$ was used to dissect the instantaneous and phase averaged velocity fields into single frequency modes that can be ranked by their dynamic significance. Only phase averaged results are repeated here. The algorithm developed in detail in Schmid ${ }^{15}$ identifies an estimate $\mathbf{S}$ of a linear mapping $\mathbf{A}$ between a temporal sequence of $\mathbf{N}$ flow snapshots from experiment $\mathbf{V}_{\mathbf{1}}^{\mathbf{N}}$, where the subscript denotes first snapshot and superscript denotes final. $\mathbf{S}=\mathbf{U}^{\mathbf{H}} \mathbf{V}_{\mathbf{2}}^{\mathbf{N}} \mathbf{W} \boldsymbol{\Sigma}^{-\mathbf{1}}$ where $\mathbf{U}$ and $\boldsymbol{\Sigma}$ are formed from a singular value decomposition on the sequence $\mathbf{V}_{\mathbf{1}}^{\mathbf{N}-\mathbf{1}}=\mathbf{U} \boldsymbol{\Sigma} \mathbf{W}^{\mathbf{H}}$. The dynamic modes $\phi_{i}=\mathbf{U y}_{\mathbf{j}}$ are found multiplying the right singular mode $\mathbf{U}$ of by the eigenvectors of $\mathbf{S}, \mathbf{y}_{\mathbf{i}}$. The growth rate and frequency of 
each mode $\phi_{j}$ are given by the eigenvalues of $\mathbf{S}, \mu_{j}$. A logarithmic mapping $\lambda_{j}=\log \mu_{j} / \Delta t$ where $\Delta t$ is the time step between frames transforms the eigenvalues such that modes with $\operatorname{Real}(\lambda)=\lambda_{r}>0$ are growing and $\lambda_{r}<0$ decay. The frequency of oscillation in $\operatorname{rad~s}^{-1}$ is given by Imaginary $(\lambda)=\lambda_{i}$. An amplitude matrix can be calculated as $\mathbf{A}=\mathbf{y}^{-\mathbf{1}} \mathbf{\Sigma} \mathbf{W}^{\mathbf{H}} \mathbf{Z}^{-\mathbf{1}}$ where $\mathbf{Z}$ is a Vandermonde matrix

$$
\mathbf{Z}=\left(\begin{array}{cccc}
z_{1}^{0} & z_{1}^{1} & \cdots & z_{1}^{N} \\
z_{2}^{0} & z_{2}^{1} & \cdots & z_{2}^{N} \\
\vdots & \vdots & \ddots & \vdots \\
z_{m}^{0} & z_{m}^{1} & \cdots & z_{m}^{N}
\end{array}\right)
$$

where $z_{j}=e^{i \lambda_{j} \Delta t}$. In practice the Vandermonde matrix can be near singular, thus numerical inversion may lead to errors. Instead $\mathbf{A}^{-\mathbf{1}}=\mathbf{Z V} \mathbf{\Sigma}^{-\mathbf{1}} \mathbf{y}$ is calculated and nearly diagonal thus the individual mode amplitude can be taken as $\mathrm{a}=1 / \operatorname{diag}\left(\mathbf{A}^{-1}\right)$.

In order to perform dynamic mode decomposition on the present data set the largest possible airfoil fixed bounding box was selected from the upper side of the airfoil in figure 4a so that the airfoil shadow does not have an effect. The extent of this box can be seen in figure 13. Due to the significant motion of the field of view around the airfoil the bounding box was limited to 1 chord length in the $\mathrm{x}$ direction and $1 / 4$ chord length in the y direction and includes the flow over the front $60 \%$ of the airfoil. The time series was sub-sampled by a factor of four $\left(\Delta t=1 / 20 \mathrm{~s}^{-1}\right)$ for numerical stability. The DMD algorithm is insensitive to non-integer numbers of periods, ${ }^{17}$ as such the data set is not truncated in time, but the full time sequence is used to achieve optimal convergence of the DMD modes.

\section{Results}

\section{A. Separation evolution}

Extruding time along the $\mathrm{Z}$ axis as in figure $4 \mathrm{~b}$ and and plotting an isocontour of vorticity (colored by velocity magnitude) allows the evolution of vortical structure in the flow to be visualized. Figure 5 shows one such isocontour over two full periods of the airfoil motion. At zero angle of attack, point A (bottom right in the figure), the vorticity contour follows the surface of the airfoil. As the airfoil pitches up and decelerates, the location of maximum velocity magnitude moves forward until the flow separates at $\alpha \sim 25$, point $\mathrm{B}$, just before maximum angle of attack and well above the static stall angle of $11.5^{\circ}$. After separation the vorticity lies in the shear layer and the velocity magnitude is nearly constant on the isocontour. Shortly before $\alpha=0$ the flow reattaches from the leading to trailing edge at point C. On the pressure side of the airfoil the vorticity again follows the airfoil surface and its location moves aft until maximum negative angle of attack point D. As the airfoil pitches back up the vorticity contour moves back toward the leading edge to the $\alpha=0$ state at point $\mathrm{A}^{\prime}$.

The first clear structural change in the vorticity isocontour, preceding separation, is the split between leading and trailing edge vorticity visible near the bottom of figure 6 at point $\beta$. Prior to $\beta$ the isocontour is relatively smooth over the entire airfoil surface. However after $\beta$ there are two distinct structures visible: one at the leading edge, with higher velocity, and one at the trailing edge with a lower velocity. As the airfoil pitches up these structures continue to diverge, the leading edge structure moving forward until separation at point B and the trailing edge structure moving aft. The trailing edge structure dissipates almost completely behind the airfoil and is no longer visible by maximum angle of attack.

A closer look at the trailing edge vorticity structure in figure 7 shows that when it first appears at point $\beta$ the structure is located at the airfoil surface. However as pitch up continues the structure quickly moves upward, away from the airfoil. The motion of this structure upwards and backwards in the airfoil frame suggests that it is quickly shed from the trailing edge and convects downstream, while the trailing edge moves away from the structure due to the pitching motion. As the airfoil pitches up, nearing separation, vorticity strengthens around the leading edge of the airfoil. Figure 8 shows that this forward growth stops when the leading edge vorticity abruptly separates at point B. Once separation occurs the leading edge vorticity begins to recede back to the suction side of the airfoil and the curvature of the vorticity contour flattens out into a shear layer aligned with the incoming flow. 


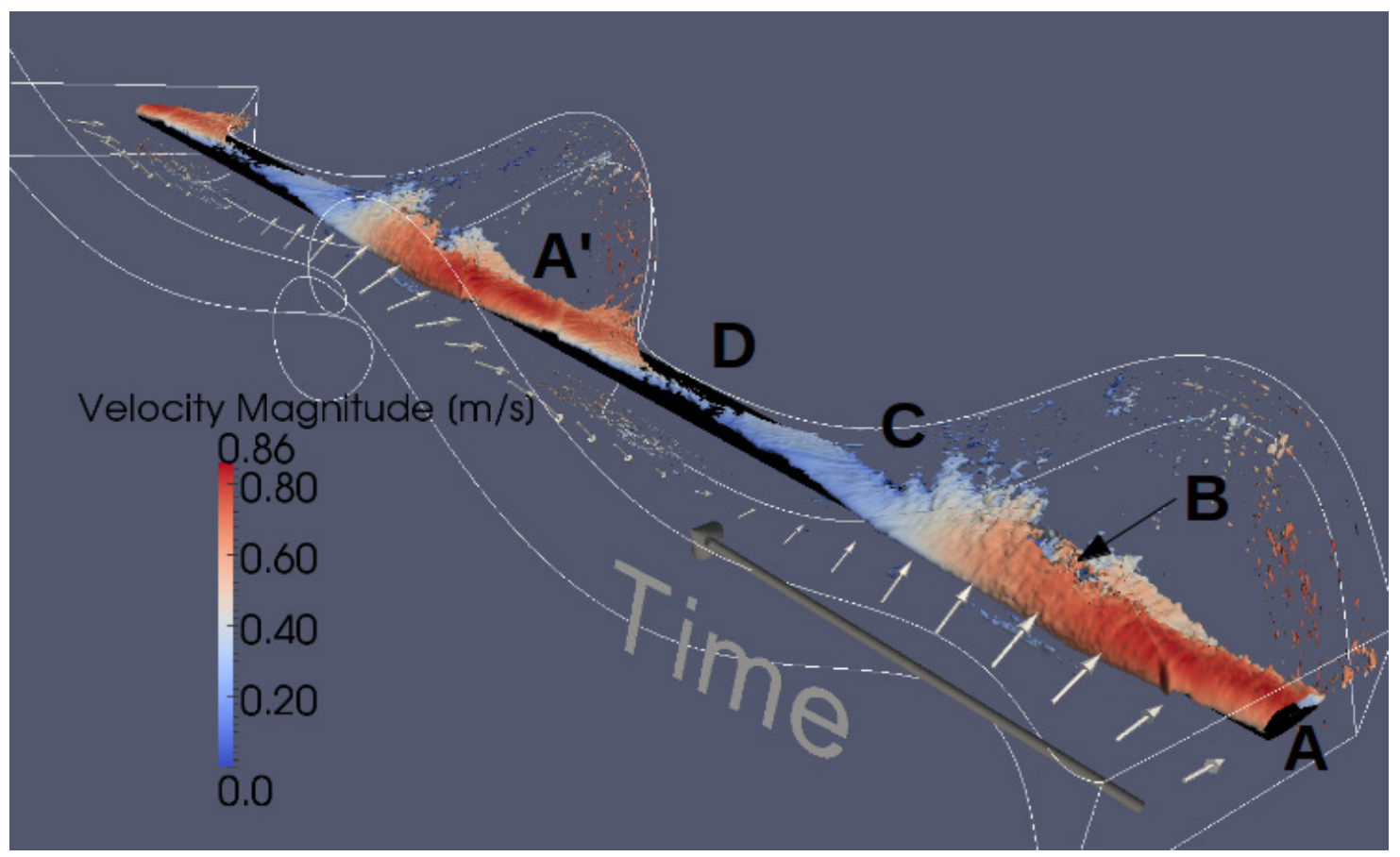

Figure 5: Vorticity isocontour colored by velocity magnitude. Two periods shown. Arrows indicate incoming angle of attack variation. A, A' at $\alpha=0, \mathrm{~B}$ at separation location, $\mathrm{C}$ at reattachment, $\mathrm{D}$ at minimum angle of attack. White lines show field of view, note that at point $\mathrm{D}$ flow at and behind the trailing edge cannot be measured.

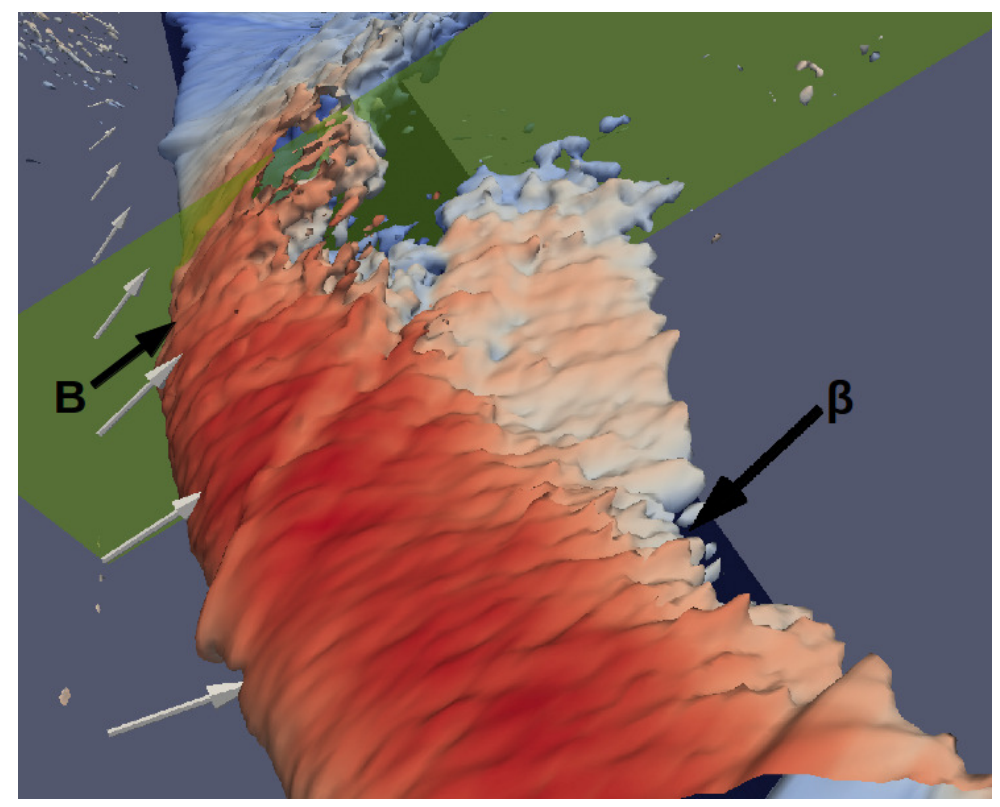

Figure 6: Vorticity isocontour leading up to separation. Trailing edge vorticity structure apperent at $\beta$. Point B indicates separation location just before maximum angle of attack (green sheet). 


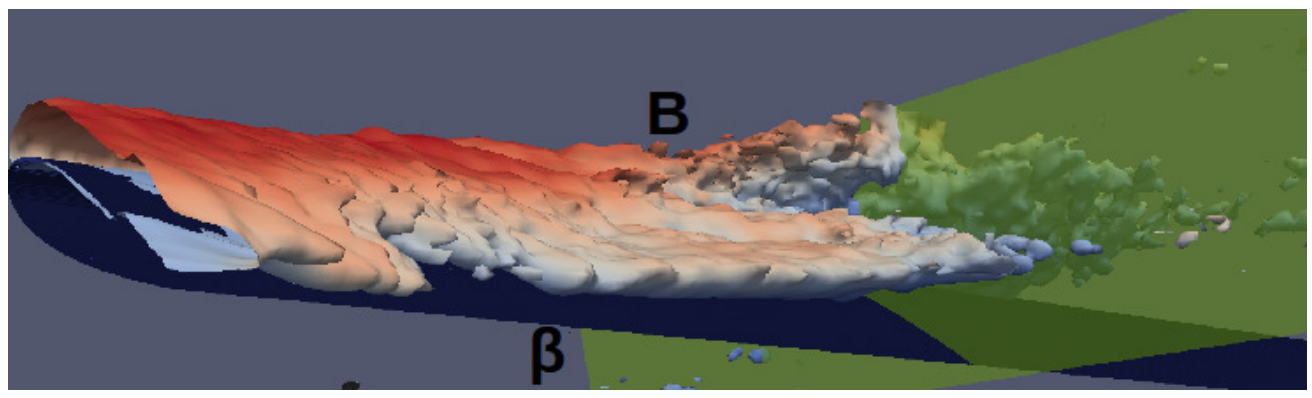

Figure 7: Vorticity isocontour from behind during pitch up process, time from left to right. Trailing edge structure appears at $\beta$ and moves up and away from the trailing edge. Maximum angle of attack indicated in green, separation at point $\mathrm{B}$.

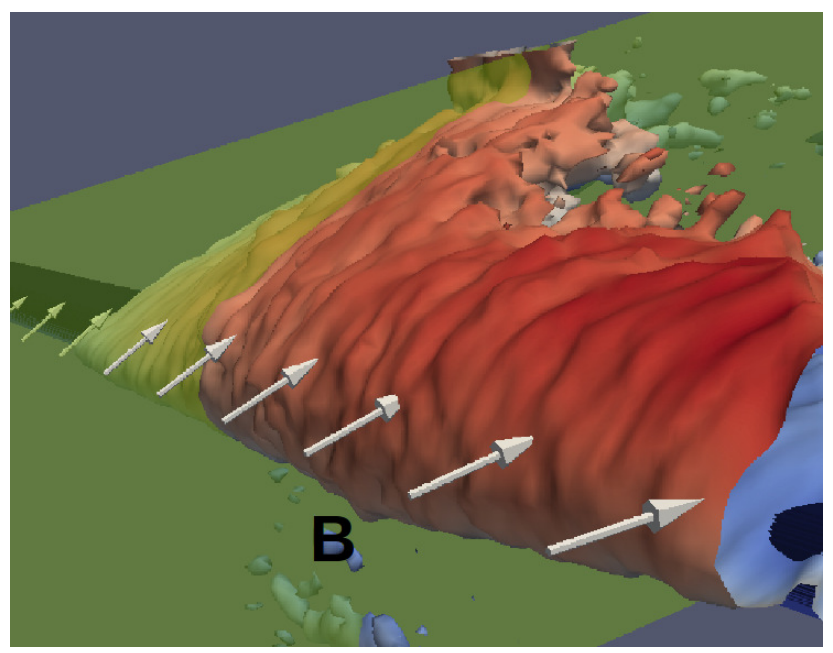

Figure 8: Leading edge vorticity isocontour during pitch up, maximum angle of attack indicated in green. Vorticity isocontour moves forward around the airfoil until separation at point B. 

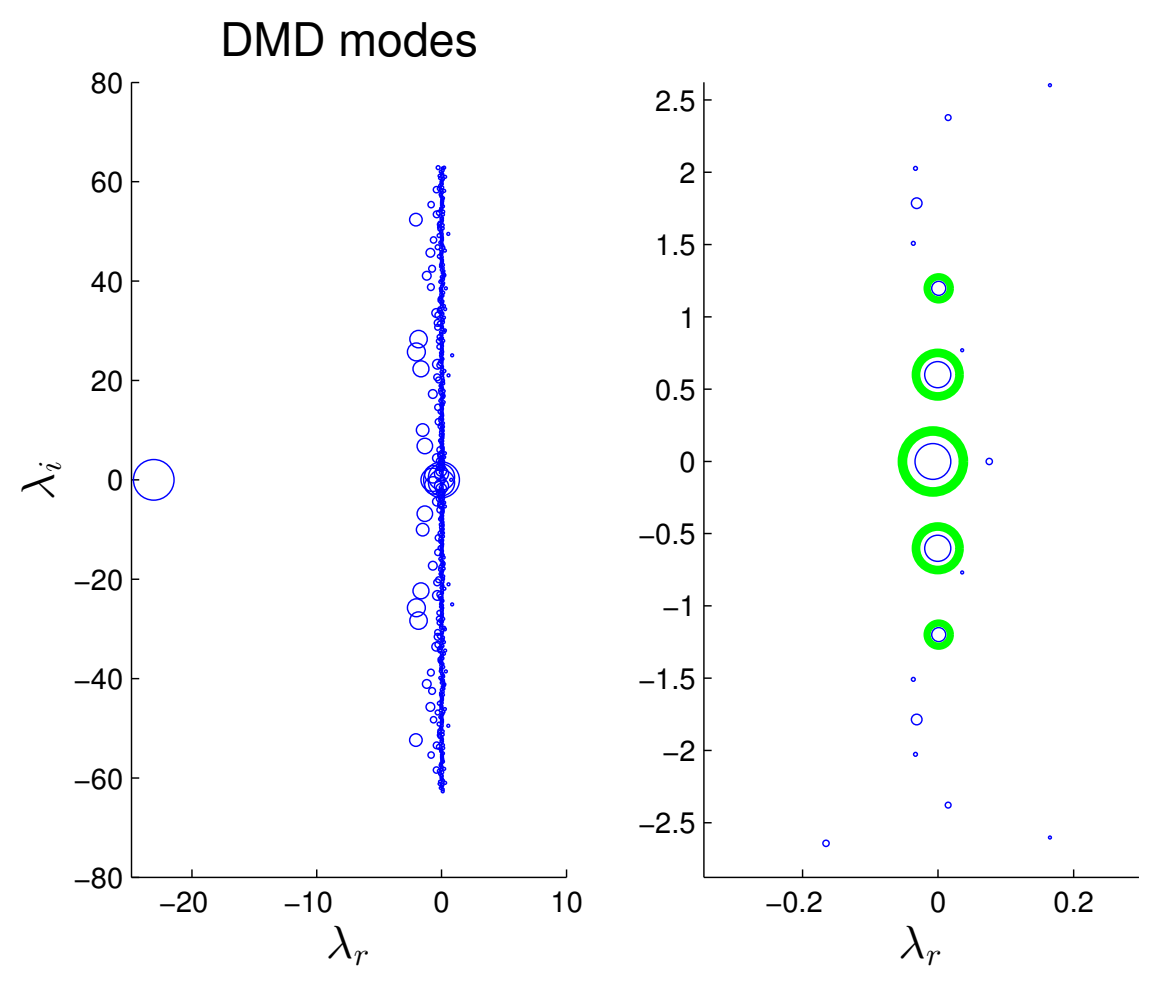

Figure 9: Modes calculated with DMD. $\lambda_{r}$ growth rate $\lambda_{i}$ frequency. Point size scaled by the magnitude of the spatial structure, a. Left full spectrum, right zoomed in on strong, non-decaying modes. Modes circled in green used for further analysis.

\section{B. Low order model}

Transformed DMD mode eigenvalues $\lambda$ are plotted in figure 9 where the $\mathrm{x}$ axis, $\lambda_{r}$, is the real part of $\lambda$ representing the modal growth rate and the y axis, $\lambda_{i}$, is the imaginary part, representing the modal frequency. As evident in figure 9 the majority of the modes cluster about the imaginary axis as is expected for phase averaged measurements. Strongly decaying modes (to the left of the imaginary axis) are not expected to be physically relevant for this data set, and are likely the results of experimental variation. Zooming in on the time constant modes with strong amplitudes as shown in the right of figure 9 shows a very strong base flow with $\lambda_{r} \approx \lambda_{i} \approx 0$, a mode pair at the pitch/surge frequency $\lambda_{i} \approx \pm 0.6$ another at $\lambda_{i} \approx \pm 1.2$ highlighted in green.

A model of the flow in an airfoil centered grid is made by combining these five dynamic modes. Qualitatively this five mode model does a good job reconstructing the data on the same grid, shown in figure 10. During pitch up, the 5 mode model captures the progression of the vorticity contour forward around the leading edge of the airfoil. In both cases the vorticity contour stops following the airfoil at point B indicating flow separation. After separation the vorticity lies in the separated shear layer as shown in section III A, but due to the small size of DMD grid away from the airfoil in the y direction this is not captured in the data analysed in the DMD model. The model however does capture the shape of the vorticity isocontour in the grid after separation as well as the reattachment. As expected the DMD representation does not include the high frequency variation apparent in the data, but models the primary behavior of the flow.

The presence of a leading edge vortex structure is identified using the $\Gamma_{1}$ and $\Gamma_{2}$ criteria developed in Graftieaux et al. 2001. ${ }^{18}$ These methods calculate a vortex center and core location respectively based on the velocity field. As such they avoid using numerical derivatives as required for methods based on the velocity gradient tensor such as the $\mathrm{Q}, \lambda_{2}$ or $\lambda_{\text {ci }}$ criteria, ${ }^{19}$ and are less sensitive to numerical noise. $\Gamma_{1}$ and $\Gamma_{2}$ at a point $\mathrm{P}$ within boundary $\mathrm{S}$ are given by

$$
\Gamma_{1}(\mathrm{P})=\frac{1}{\mathrm{~S}} \int_{\mathrm{M} \in \mathrm{S}} \frac{\mathrm{PM} \times \mathrm{U}_{\mathrm{M}} \cdot \mathrm{z}}{\|\mathrm{PM}\| \cdot\left\|\mathrm{U}_{\mathrm{M}}\right\|} \mathrm{dS}=\frac{1}{\mathrm{~S}} \int_{\mathrm{S}} \sin \left(\theta_{\mathrm{M}}\right) \mathrm{dS}
$$




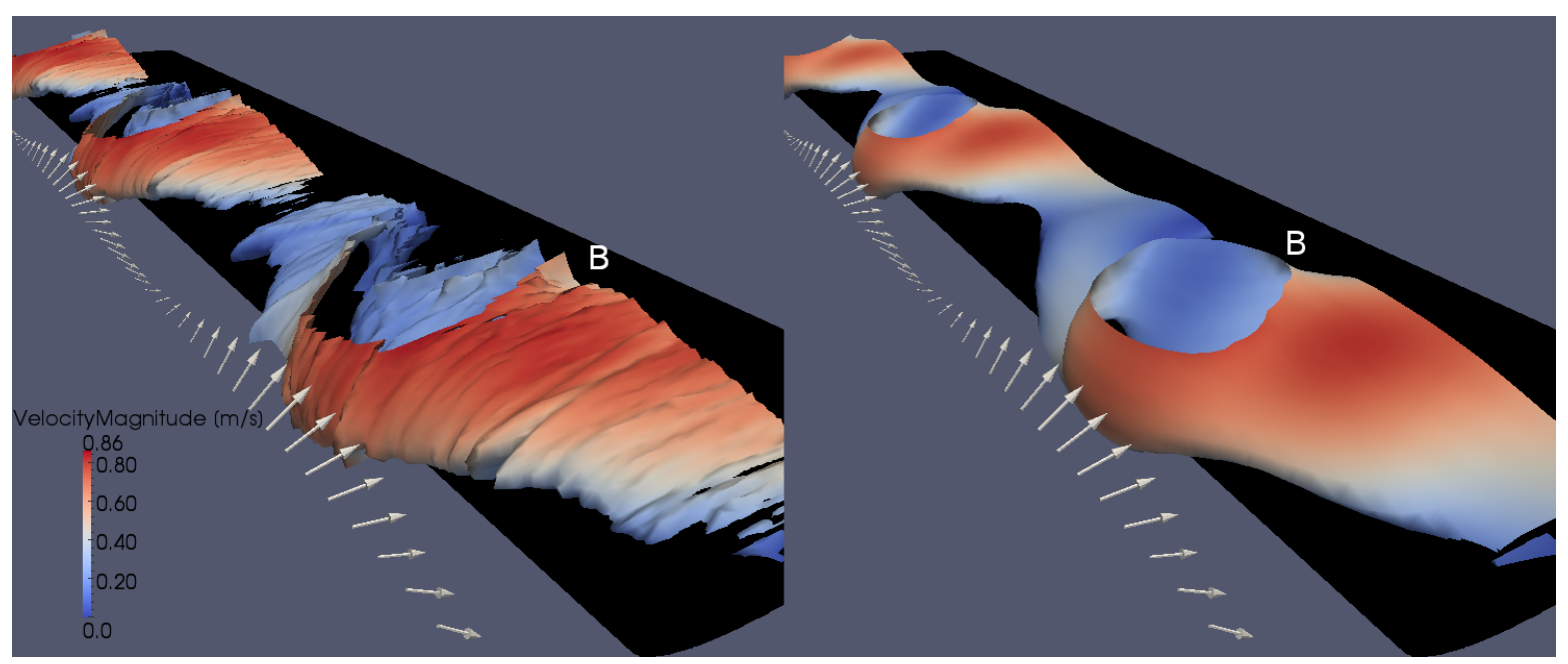

Figure 10: Isocontour of vorticity (colored by velocity magnitude) of data (left) and 5 mode DMD reconstruction (right) for entire data set. Reconstruction captures primary behavior of flow.

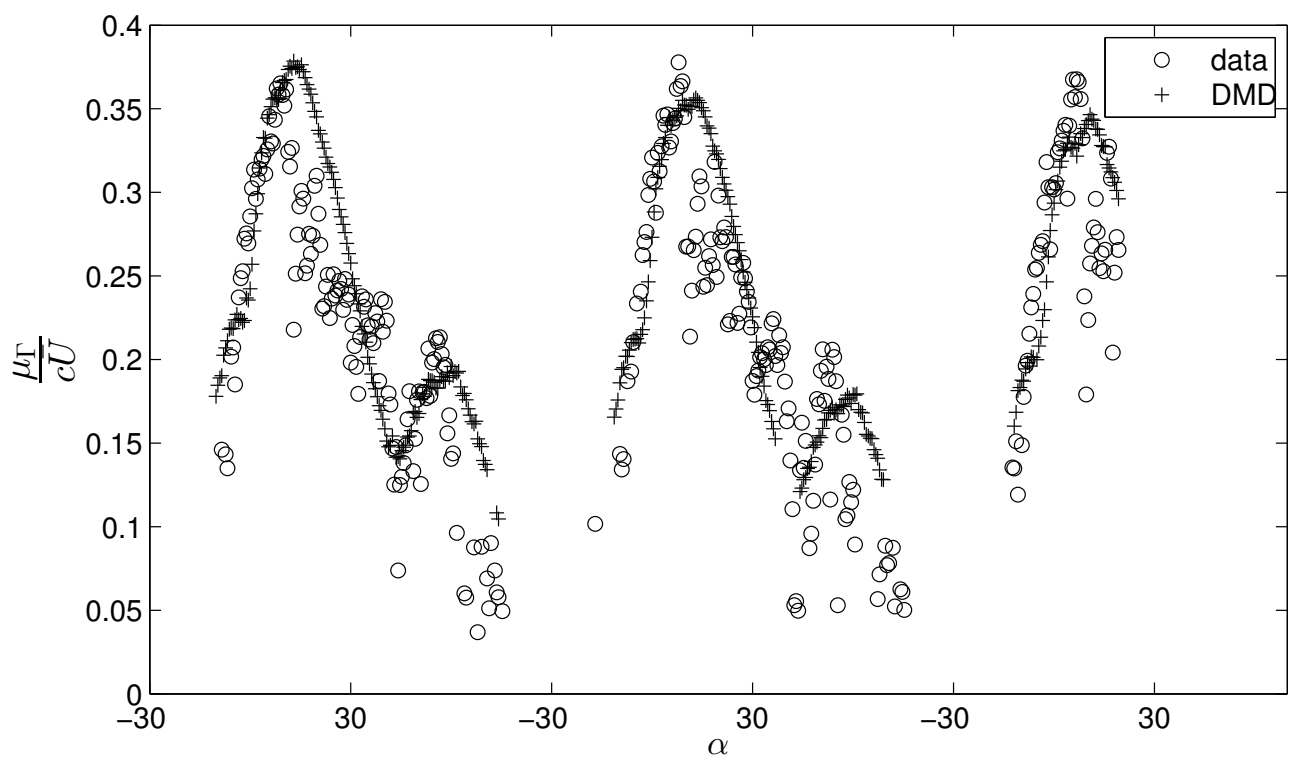

Figure 11: Normalized circulation within leading edge vortex of 5 mode DMD model (+) and data (o). Plotted against airfoil angle of attack, $\alpha$. 


$$
\Gamma_{2}(\mathrm{P})=\frac{1}{\mathrm{~S}} \int_{\mathrm{M} \in \mathrm{S}} \frac{\mathrm{PM} \times\left(\mathrm{U}_{\mathrm{M}}-\mathrm{U}_{\mathrm{P}}\right) \cdot \mathrm{z}}{\|\mathrm{PM}\| \cdot\left\|\left(\mathrm{U}_{\mathrm{M}}-\mathrm{U}_{\mathrm{P}}\right)\right\|} \mathrm{dS}
$$

where $\mathrm{S}$ is the area around $\mathrm{P}$ and $\mathrm{M}$ lies in $\mathrm{S}$. $\mathrm{PM}$ is the radius vector from $\mathrm{P}$ to $\mathrm{M}, \mathrm{U}_{\mathrm{M}}$ is the velocity at point $\mathrm{M}, \theta_{\mathrm{M}}$ is the angle between $\mathrm{PM}$ and $\mathrm{U}_{\mathrm{M}}$ and $\mathrm{U}_{\mathrm{P}}$ is the local convection velocity at $\mathrm{P}$. The maximum value of $\Gamma_{1}$ gives the location of the vortex core, and values of $\left|\Gamma_{2}\right|>2 / \pi$ locate the vortex boundary where rotation locally dominates shear.

For the present data a circular stencil of with a radius of 3 grid cells was generated with MATLAB and summed to approximate the integral. A threshold of $\Gamma_{1}>.7$ was used to indicate the existence of a vortex. The circulation $\left(\mu_{\Gamma}\right)$ within the vortex boundary $\Gamma_{2}>2 / \pi$ was calculated and normalized by the mean velocity $\overline{\mathrm{U}}$ and the chord length $\mathrm{c}$ for both the 5 mode DMD model and the original data. The results are plotted in figure 11. Clearly the 5 mode DMD model does a very good job of capturing the LEV circulation, capturing both the shape and the magnitude. The peak in circulation appears just before separation $\alpha \sim 25^{\circ}$ when most of the vorticity is contained within the vortex at the leading edge (figure 12a). After separation vorticity follows the shear layer extending outside the DMD domain, and circulation decreases figure(12b). The second peak that appears in both the data and DMD reconstruction occurs near reattachment when rotational flow once again begins to follow the airfoil surface, and is contained within the vortex boundary (figure 12c).

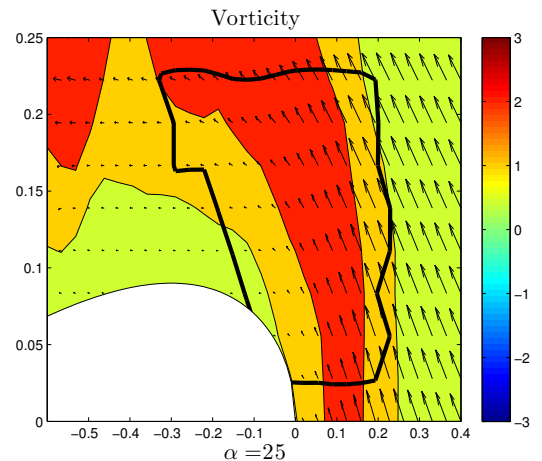

(a) Flow before separation on pitch up. Strong vorticity exists within leading edge vortex boundary.

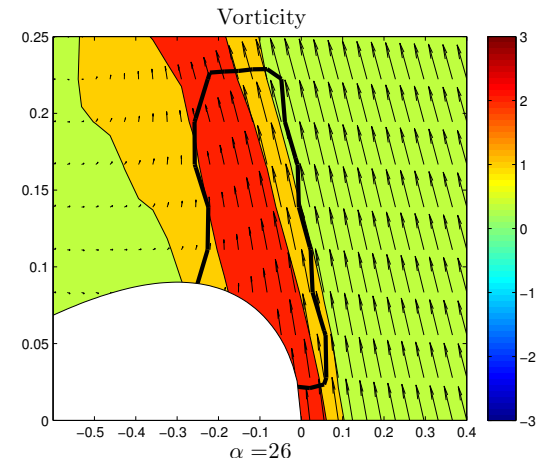

(b) Flow after separation on pitch down. Shear layer extends outside measurement domain.

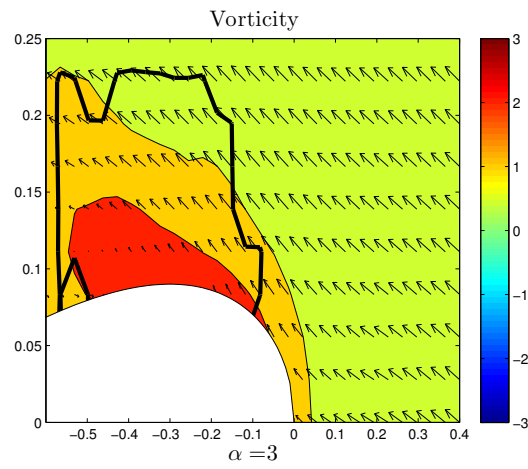

(c) Flow after reattachment on pitch down. Circulation back within vortex boundary.

Figure 12: Velocity (vectors), vorticity (filled contours), and $\Gamma_{2}=2 / \pi$ vortex boundary (black line) of full velocity field contained in DMD measurement grid.

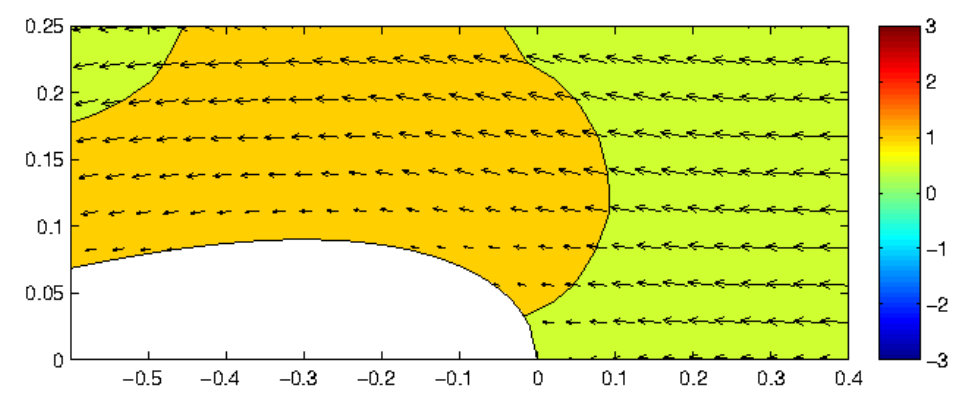

Figure 13: Velocity (vectors) and vorticity (contour) plots of time constant base flow from single DMD mode. Incoming flow and diffuse vorticity from flow curvature around the airfoil captured.

The mean mode is shown in figure 13. It represents a time constant base flow with approximately 0 angle of attack and diffuse vorticity due to the rotation of the flow around the airfoil body. The first complex conjugate pair mode is plotted in figure 14 at various points in the airfoil cycle. This mode includes the velocity and angle of attack variation caused by the pitch and surge motion. It provides positive $\mathrm{x}$ velocity 
during surge forward (figures 14a-14c), negative during surge back (figures 14d-14e), positive y velocity for positive $\alpha$ (figures 14b-14c) and negative for negative $\alpha$ (figure 14e).

Increased positive vorticity during pitch up and negative vorticity during pitch down suggests the presence of vortex structure in the mode with counter clockwise rotation during pitch up and clockwise during pitch down. In figure 15 the first mode is plotted with the instantaneous surge velocity subtracted, making this vortical structure in the mode much clearer. Visible in figure 15a is the formation of a counter-clockwise vortex above the leading edge. As the airfoil is pitched up this vortex convects downstream, as seen in figure 15b. Congruent with a periodic process, on pitch down a vortex of the opposite direction is formed and convects downstream on the pressure side of the airfoil. This behavior suggests a primary separation mode, containing a vortex that grows at the leading edge during the pitch up and convects downstream lifting the streamline from the surface and leading separation. On pitch down the opposite signed vortex lags the reattachment. This process is shown schematically in figure 16 .

The behavior of the dynamic mode at $\Omega=1.2 \mathrm{rad} \mathrm{s}^{-1}$ is shown in figure 17 . The period of this mode is half that of the airfoil motion, thus a full mode cycle takes place on the suction side of the airfoil $\alpha \geq 0$. A positive direction vortex is seen to nucleate at the leading edge and convect downstream along the shear layer, figures $17 \mathrm{a}, \mathrm{b}$ and $\mathrm{c}$. This vortex lags separation led by the primary separation mode and strengthens the shear layer. During pitch down the corresponding negative vortex pushes the streamline back toward the airfoil surface and leads reattachment.

The phase relationship between primary and secondary modes provides the different behavior on the pressure and suction side of the airfoil. A plot of mode strength normalized by the strength of the primary stall mode is plotted in fig 19. On the suction side the primary and secondary modes interact constructively. Maximum primary mode strength occurs at $\alpha=0$, leads separation and is enhanced by the secondary mode. During pitch down the secondary mode peaks first at $\alpha=15^{\circ}$, leading reattachment, and is enhanced by the primary separation mode. On the pressure side of the airfoil primary and secondary separation modes act in the opposite direction and the effect of each mode counters the other. Destructive interference between these two modes results in a weaker combined effect on the base flow, maintaining attached flow on the pressure side, while angle of attack and velocity are preserved.

\section{Summary and conclusions}

The flow over a model of the blade of a vertical axis wind turbine (VAWT) was investigated experimentally to explore dynamic separation resulting in unsteady loading on the turbine. A NACA 0018 airfoil at a mean Reynolds number of $10^{5}$ was pitched between $-30^{\circ} \leq \alpha \leq 30^{\circ}$ and surged sinusoidally such that $\frac{\mathrm{U}_{\max }-\mathrm{U}_{\text {min }}}{\mathrm{U}}=.9$ at a reduced frequency $\mathrm{k}=0.12$ to match the angle of attack and velocity variation of an industrial VAWT at tip speed ratio $\eta=2$. Time resolved particle image velocimetry (PIV) was used to measure the flow field over multiple periods of the airfoil motion. Results of multiple experiments, ensemble averaged, based on the phase of the airfoil were presented. Vorticity contours were shown to follow the airfoil surface, up to very high angle of attack $\alpha \sim 25^{\circ}$ indicating attached flow. Above $\alpha=25^{\circ}$ the flow separates and the vorticity follows the shear layer, until reattachment on pitch down as the airfoil approaches zero angle of attack.

A low order model of the phase averaged flow over the leading edge was made using five modes identified using dynamic mode decomposition (DMD). These modes have negligible growth rate and include frequencies at one and two times the frequency of airfoil motion as well as a time constant mode. This model is able to capture the growth of vorticity over the leading edge of the airfoil, as well as the separation, shear layer formation and reattachment. The $\Gamma_{1}$ and $\Gamma_{2}$ vortex identification tool were utilized to identify a leading edge vortex structure. Circulation within the LEV was well represented by the DMD model, accurately capturing the growth of the vortex and its subsequent separation from the leading edge.

The time history of the DMD mode pairs at each frequency was analysed. The mode pair at the airfoil frequency captures the $\mathrm{x}$ and $\mathrm{y}$ velocity variation necessary to create the free stream velocity and angle of attack variation from the pitch and surge motion. Additionally over the airfoil this mode includes a vortex structure that acts to lift the streamline away from the airfoil surface, effectively leading separation. The second mode pair, at twice the airfoil frequency, includes a similar vortex structure. During pitch up this vortex has the same sense of rotation as the first mode, lagging separation, and strengthening the shear layer. On pitch down the vortex is in the opposite direction, pushing the flow back toward the airfoil surface and leading reattachment. On the pressure side of the airfoil $(\alpha<0)$, these modes act in opposing directions 


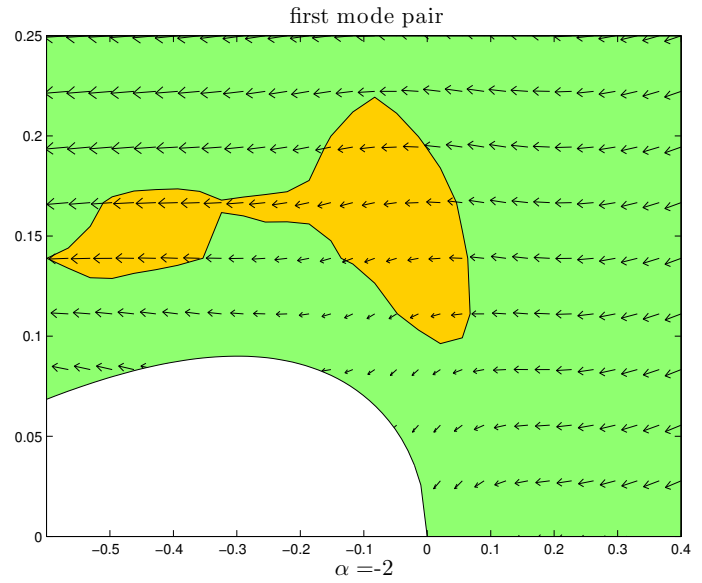

(a) First DMD conjugate pair $\alpha=-2$ on pitch up.

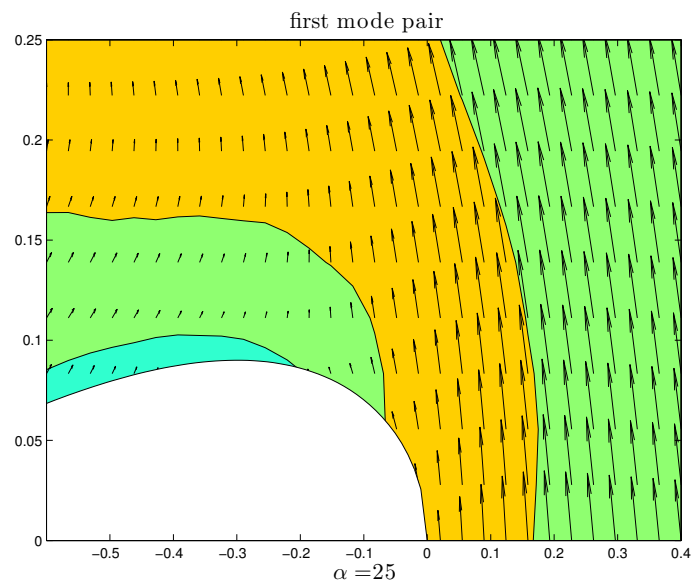

(c) First DMD conjugate pair $\alpha=25$ on pitch up.

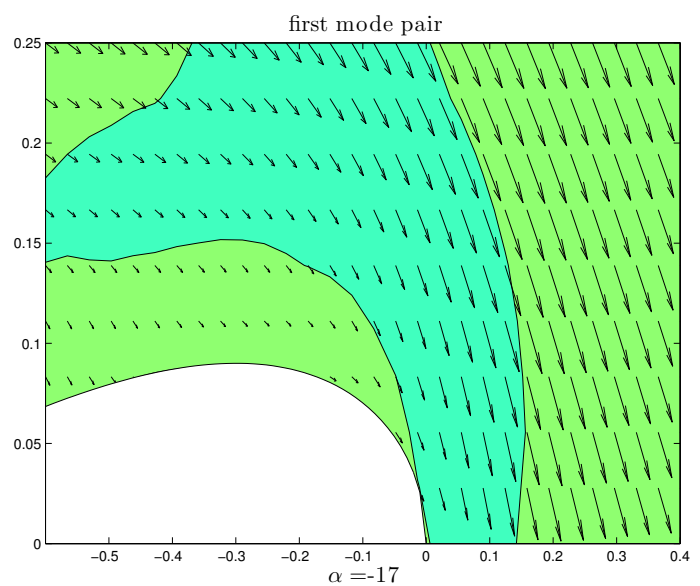

(e) First DMD conjugate pair $\alpha=-17$ on pitch down.

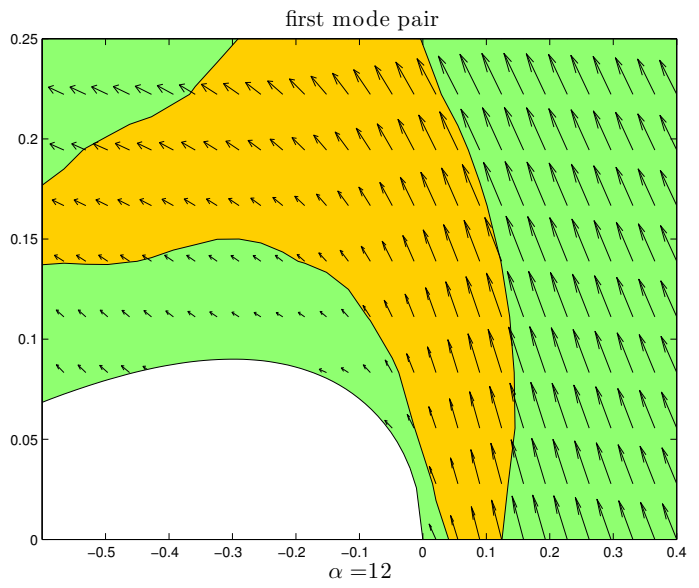

(b) First DMD conjugate pair $\alpha=12$ on pitch up.

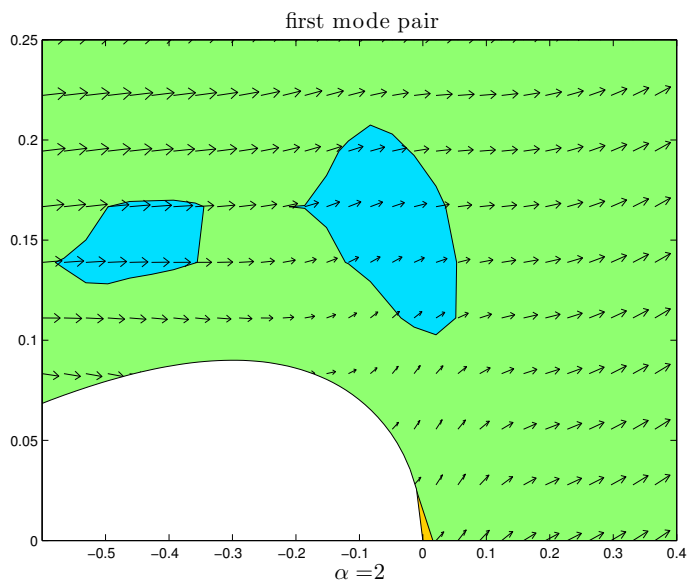

(d) First DMD conjugate pair $\alpha=2$ on pitch down.
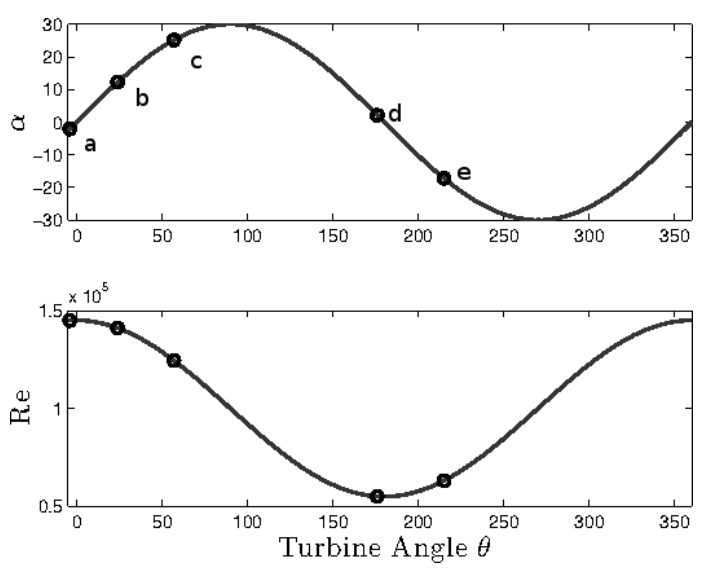

(f) Location of each plot a-e on pitch surge cycle.

Figure 14: Velocity (vector) and vorticity (contour) plots of first DMD conjugate pair at pitch surge frequency $\Omega=.6 \mathrm{rad} / \mathrm{s}$. Freestream angle of attack and velocity variation provided by this mode. Contour levels set at $1 / 3$ of maximum vorticity, cold colors indicate negative vorticty, warm positive. 


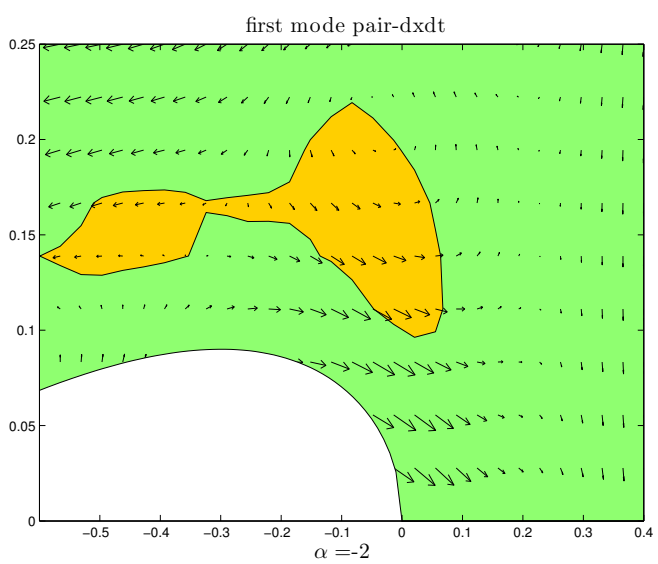

(a) First DMD conjugate pair without surge component $\alpha=-2$ on pitch up.

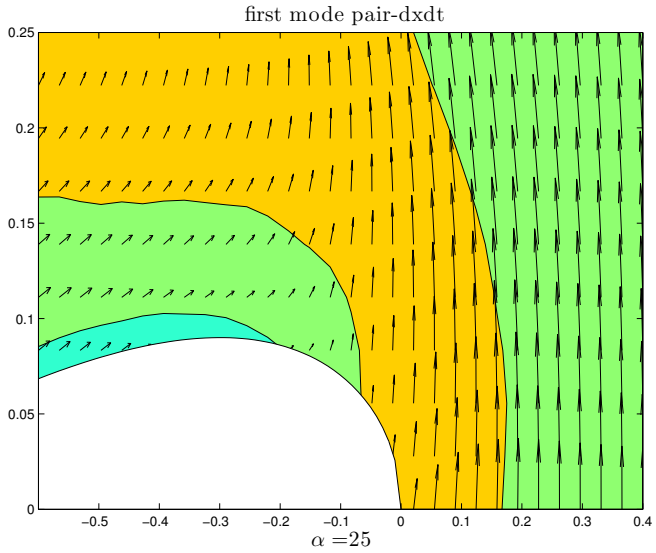

(c) First DMD conjugate pair without surge component $\alpha=25$ on pitch up.

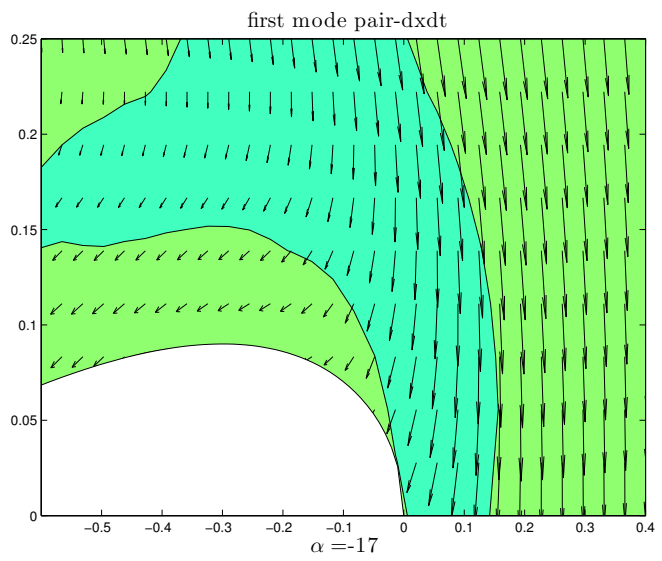

(e) First DMD conjugate pair without surge component $\alpha=-17$ on pitch down.

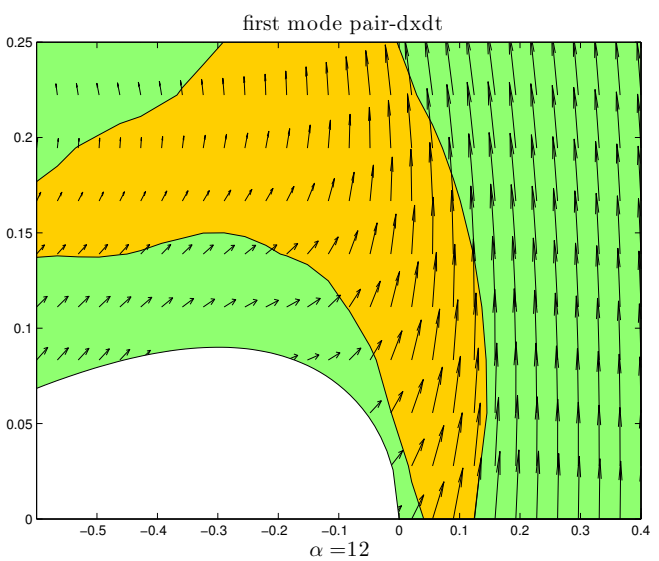

(b) First DMD conjugate pair without surge component $\alpha=12$ on pitch up.

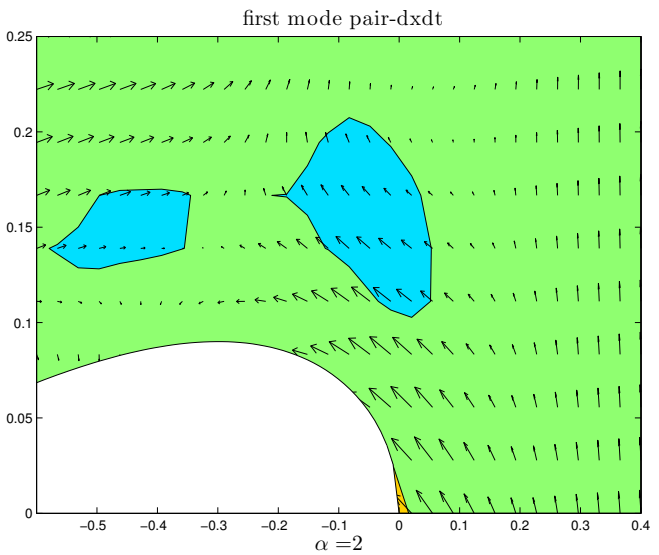

(d) First DMD conjugate pair without surge component $\alpha=2$ on pitch down.
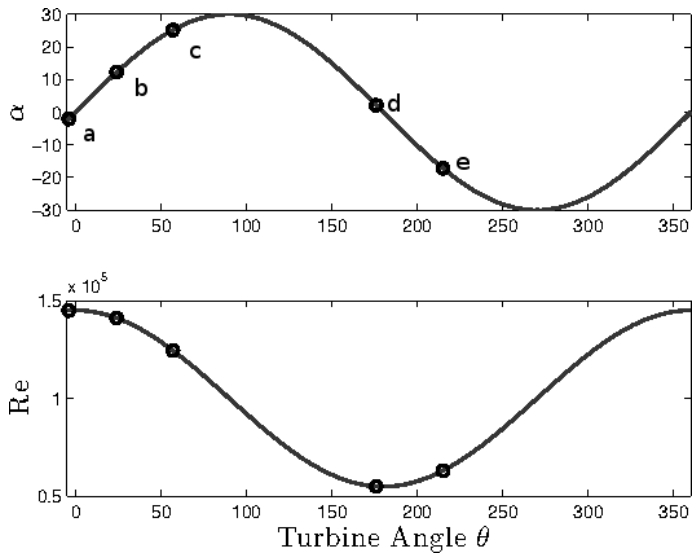

(f) Location of each plot a-e on pitch surge cycle.

Figure 15: Velocity and vorticity plots of first DMD conjugate pair at pitch/surge frequency $\Omega=.6 \mathrm{rad} / \mathrm{s}$ with the freestream variation from surge removed. Vortex structure at leading edge apparent in figures a and e. Contour levels set at $1 / 3$ of maximum vorticity, cold colors indicate negative vorticty, warm positive. 

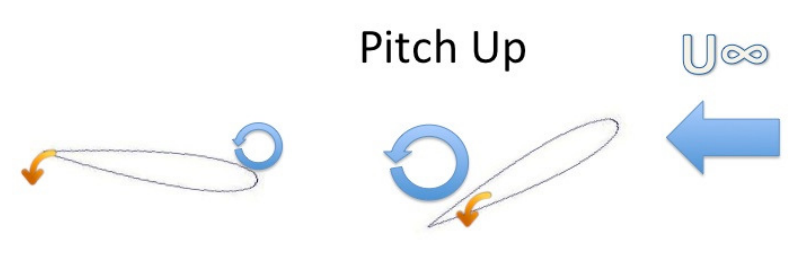

\section{Pitch Down}
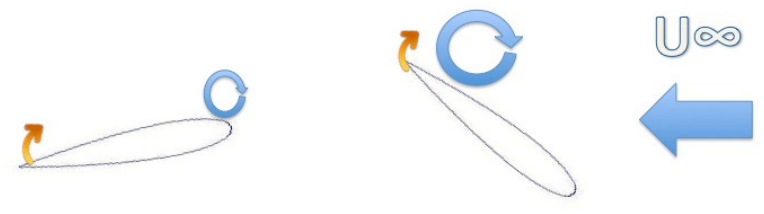

Figure 16: Schematic of the model of a primary separation DMD mode at the airfoil frequency. Counterclockwise rotating vortex appears on pitch up, convecting downstream and leading separation. Clockwise vortex forms after reattachment, and enforces attached flow on pressure side.

keeping the flow attached.

Future research will investigate vortex shedding and the development of vortex structure at the trailing edge, as well as how it interacts with the leading edge flow dynamics. Additionally, instantaneous, non-phase averaged, measurements will be explored to investigate flow structure not exactly coupled to the phase of the airfoil motion, and to discover finer detail in the flow.

\section{Acknowledgements}

This work was supported by the Gordon and Betty Moore Foundation through grant GBMF\#2645 to the California Institute of Technology. The authors thank Professor Peter J. Schmid for his assistance in implementing the dynamic mode decomposition algorithm. Professor Morteza Gharib for the use of the free surface water channel facility, Hsieh-Chen Tsai and Professor Tim Colonius for discussion on the Coriolis effect and Professor John Dabiri for his insight from VAWT field research. 


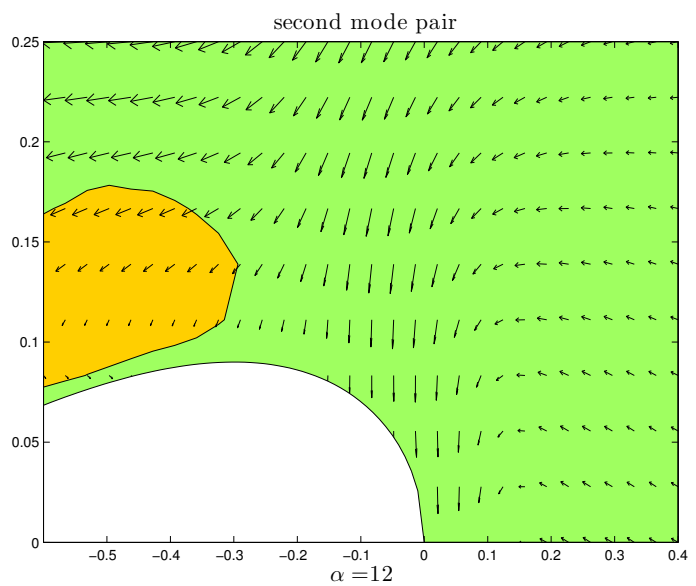

(a) Second DMD conjugate pair $\alpha=12$ on pitch up.

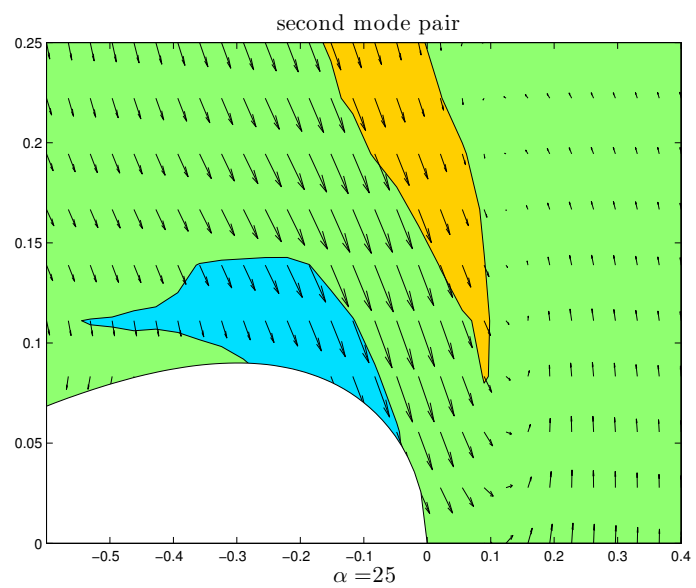

(c) Second DMD conjugate pair $\alpha=25$ on pitch up.

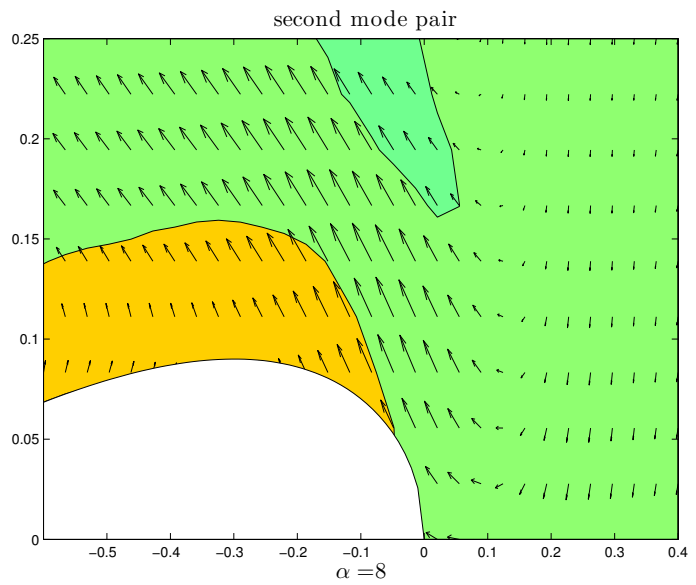

(e) Second DMD conjugate pair $\alpha=-8$ on pitch down.

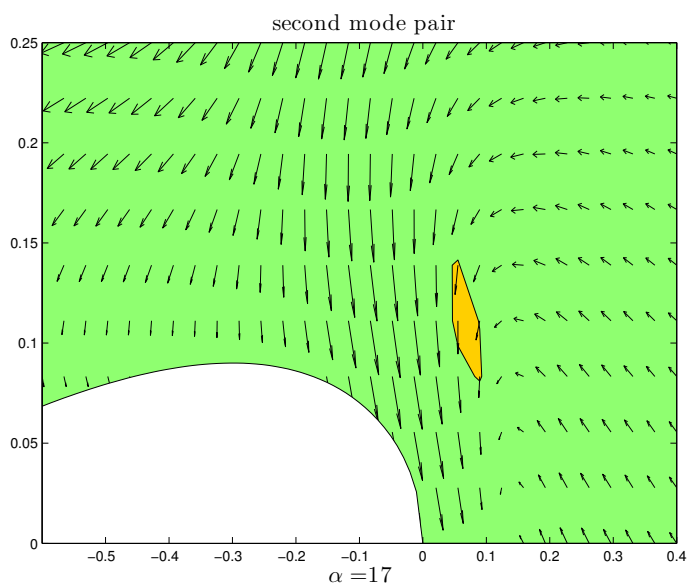

(b) Second DMD conjugate pair $\alpha=17$ on pitch up.

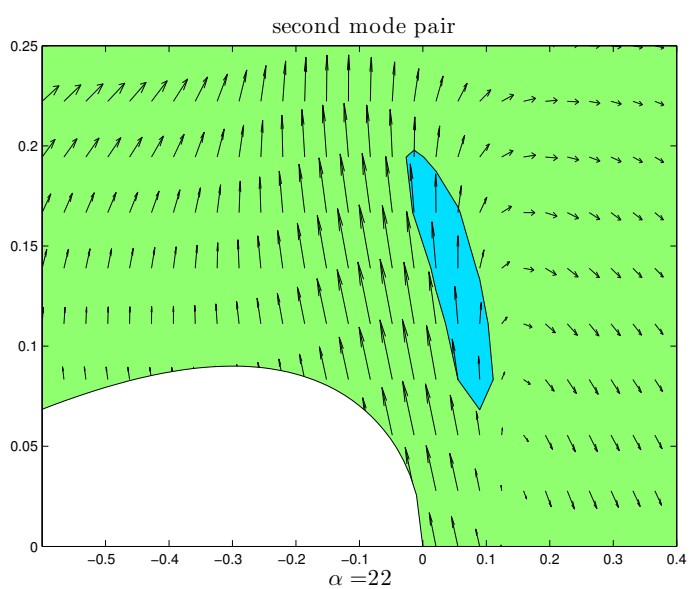

(d) Second DMD conjugate pair $\alpha=22$ on pitch down.
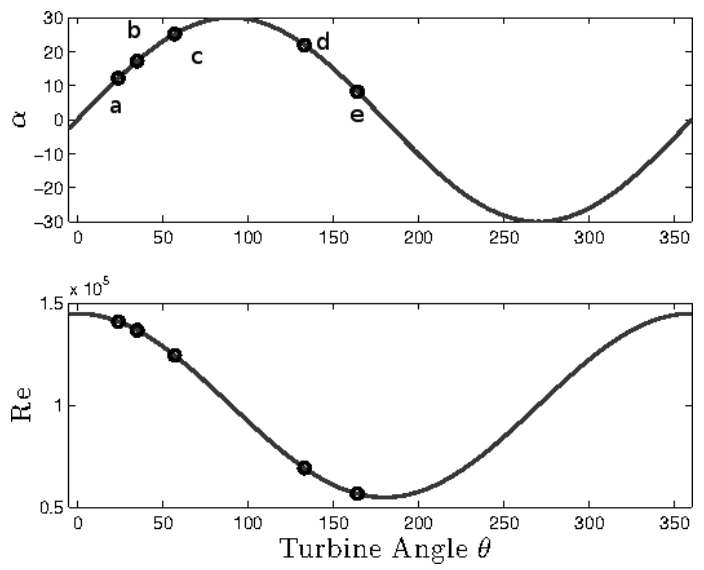

(f) Location of each plot a-e on pitch surge cycle.

Figure 17: Velocity and vorticity plots of second DMD conjugate pair at twice pitch/surge frequency $\Omega=1.2$ $\mathrm{rad} / \mathrm{s}$. Contour levels set at $1 / 3$ of maximum vorticity, cold colors indicate negative vorticty, warm positive. 

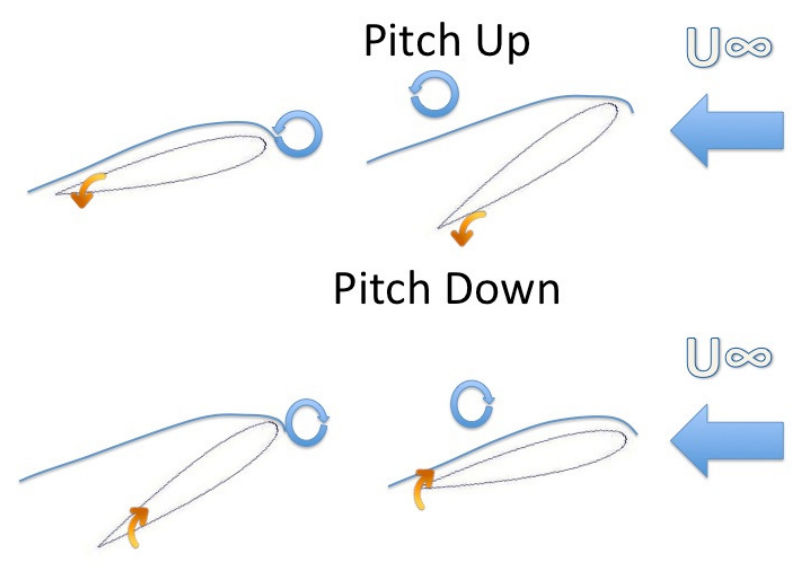

Figure 18: Schematic of the model of a secondary stall mode at twice the airfoil frequency. During pitch up a counter-clockwise vortex forms and convects along the separated shear layer, while during pitch down a clockwise vortex forms leading the reattachment process.

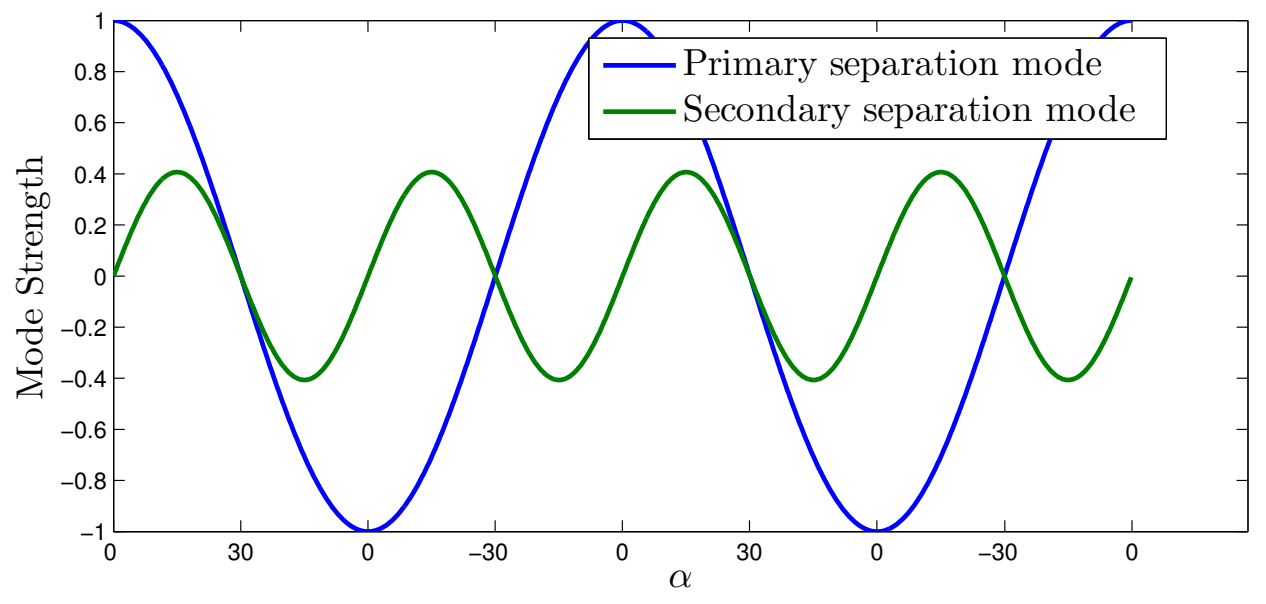

Figure 19: Primary and secondary separation mode strengths over 2 airfoil cycles. Modes interact constructively on the suction side of airfoil $(0 \leq \alpha \leq 30)$ and destructively on the pressure side $(0 \geq \alpha \geq-30)$. 


\section{References}

1 US Energy Information Administration, "Electric power monthly," Tech. Rep. DOE/EIA-0226, US energy information administration, April 2012.

2 Hau, E., Wind turbines: fundamentals, technologies, application, economics, Springer, Berlin, third, translated edition ed., 2013.

3 Dabiri, J. O., "Potential order-of-magnitude enhancement of wind farm power density via counter-rotating vertical-axis wind turbine arrays," J. Renew. Sustain. Energy, Vol. 3, No. 4, 2011.

${ }^{4}$ Greenblatt, D., Harav, A. B., and Mueller-Vahl, H., "Mechanism of dynamic stall control on a vertical axis wind turbine," 51st AIAA Aerospace Sciences Meeting, 2013.

5 Islam, M., Ting, D. S.-K., and Fartaj, A., "Desirable airfoil features for smaller-capacity straight-bladed VAWT," Wind Eng., Vol. 31, No. 3, 2007, pp. 165-196.

${ }^{6}$ Carr, L. W., "Progress in analysis and prediction of dynamic stall," J. Aircraft, Vol. 25, No. 1, 1988.

7 Simão Ferreira, C. J., Bijl, H., van Bussel, G., and van Kuik, G., "Simulating dynamic stall in a 2D VAWT: Modeling strategy, verification and validation with particicle image velocimetry data," J. Physics: Conference Series, Vol. 73, 2007.

8 Simão Ferreira, C. J., van Bussel, G., and van Kuik, G., "2D PIV visualization of dynamic stall on a vertical axis wind turbine," $45^{\text {th }}$ AIAA Aerospace Sciences Meeting and Exhibit / ASME Wind Energy Symposium, 2007.

9 Simão Ferreira, C., van Kuik, G., van Bussel, G., and Scarano, F., "Visualization by PIV of dynamic stall on a vertical axis wind turbine," Exp. Fluids, Vol. 46, No. 1, Aug. 2009, pp. 97-108.

10 Simão Ferreira, C. J., A, V. Z., Bijl, H., van Bussel, G., and van Kuik, G., "Simulating dynamic stall in a two-dimensional vertical axis wind turbine: verification and validation with particle image velocimetry data," Wind Energ., Vol. 13, 2010.

11 Jones, A. R. and Babinsky, H., "Unsteady lift generation on rotating wings at low Reynolds number," $J$. Aircraft, Vol. 47, No. 3, 2010, pp. 1013-1021.

12 Mulleners, K. and Raffel, M., "The onset of dynamic stall revisited," Exp. Fluids, Vol. 52, 2012.

13 Mulleners, K. and Raffel, M., "Dynamic stall development," Exp. Fluids, Vol. 54, 2013.

14 Windspire, "Standard wind unit 1.2kW," Accessed 11/2013.

15 Schmid, P. J., "Dynamic mode decomposition of numerical and experimental data," J. Fluid Mech, Vol. 656, 2010.

16 Brent, S., "AWEA Standard Update," 2009.

17 Chen, K. K., Tu, J. H., and Rowley, C. W., "Variants of dynamic mode decomposition: boundary condition, Koopman, and Fourier analyses," J. Nonlinear Sci., Vol. 22, 2012.

18 Graftieaux, L., Michard, M., and Grosjean, N., "Combining PIV, POD and vortex identification algorithms for the study of unsteady turbulent swirling flows," Meas. Sci. Technol., Vol. 12, 2001.

19 Chakraborty, P., Balachandar, S., and Adrian, R. J., "On the relationships between local vortex identification schemes," J. Fluid Mech., Vol. 535, July 2005, pp. 189-214. 